\title{
Data treatment and data storage at the BioSAXS beamline TPS 13A
}

\author{
O. Shih ${ }^{1}$, C.-J. Su${ }^{1}$, Y.-Q. Yeh${ }^{1}$, K.-F. Liao ${ }^{1}$, J.-W. Chang ${ }^{1}$, C.-A. Wang ${ }^{1}$, W.-R. Wu ${ }^{1}$, and U.-S. Jeng ${ }^{1,2}$ \\ ${ }^{I}$ National Synchrotron Radiation Research Center, Hsinchu Science Park, Hsinchu 30076, Taiwan, \\ ${ }^{2}$ Department of Chemical Engineering, National Tsing Hua University, Hsinchu 30013, Taiwan
}

shih.orion@nsrrc.org.tw

The Taiwan Photon Source (TPS) 13A biological small-angle X-ray scattering (SAXS) beamline at the National Synchrotron Radiation Research Center was recently opened to users. The beamline is designed for probing biological structures and kinetics in wide length and time scales, from angstrom to micrometer and from microsecond to minutes. A 4-m IU24 undulator provides high flux X-rays in the energy range of 4.0 to $23.0 \mathrm{keV}$. $\mathrm{MoB}_{4} \mathrm{C}$ double-multilayer and $\mathrm{Si}-(111)$ double-crystal monochromators $(\mathrm{DMM} / \mathrm{DCM})$ are combined on the same rotating platform for a smooth transition from high flux beams $\left(\sim 4 \times 10^{14}\right.$ photons $\left./ \mathrm{s}\right)$ to a high-energy-resolution beam $\left(\Delta E / E \sim 1.5 \times 10^{-4}\right)$. USAXS and microbeam modes are also available through a series of carefully designed optical components. An X-ray detecting system comprising two in-vacuum detectors was designed to perform synchronized small- and wide-angle X-ray scattering data collections at the endstation.

TPS 13A beamline adopts the Experimental Physics and Industrial Control System (EPICS) [1] for integrated controls of the hardware and software, including all motors of the optical components and their corresponding sensing and cooling systems. Communication among local systems was achieved via defined process variables (PVs) provided by the EPICS Input/Output Controller (IOC) program. We have integrated two main clients of PVs: (1) Control-System Studio (CSS) GUI [2], and (2) command-line system SPEC for beamline control. The data acquisition on TPS 13A is based on a synchronous operation of all detectors, ms-shutter, and intensity monitors. With the high frame rate of the detectors and a large number of pixels, a typical protein solution SEC-SAXS experiment can generate a few GB of data. The data storage, remote data access, and data treatment problems become essential if the user community continues to grow and even more critical for future high-throughput screening applications. Here we collaborate with Academia Sinica Grid-computing Center to create an online platform called Distributed Cloud Operating System (DiCOS)-BioSAXS platform (https://bioswan.twgrid.org/). It provides TPS 13A BioSAXS beamline users a friendly interface to access their experimental data, analyze data, and submit SAXS simulation jobs.

[1] http://www.aps.anl.gov/epics/ or Dalesio, L. R., Kraimer, M. R., and Kozubal, A. J. (1991) ICALEPCS. 91.

[2] Hatje, J., Clausen, M., Gerke, C., Moeller, M., and Rickens, H. (2007). ICALEPCS MOPB03.

Keywords: BioSAXS; Data treatment; Data archiving; Grid-computing 